\title{
Perspective
}

PERSPECTIVE Actualité en histoire de l'art

2| 2007

La Grande-Bretagne/Période moderne

\section{Sélection internet}

\section{Olivier Bonfait}

\section{OpenEdition}

\section{Journals}

Édition électronique

URL : http://journals.openedition.org/perspective/3826

DOI : 10.4000/perspective.3826

ISSN : 2269-7721

Éditeur

Institut national d'histoire de l'art

Édition imprimée

Date de publication : 30 juin 2007

Pagination : 326-330

ISSN : 1777-7852

\section{Référence électronique}

Olivier Bonfait, « Sélection internet », Perspective [En ligne], 2 | 2007, mis en ligne le 31 mars 2018 consulté le 01 octobre 2020. URL : http://journals.openedition.org/perspective/3826 ; DOI : https:// doi.org/10.4000/perspective.3826

Ce document a été généré automatiquement le 1 octobre 2020. 


\title{
Sélection internet
}

\author{
Olivier Bonfait
}

1 Cette liste permet de présenter des sites ou des rubriques internet importants par les institutions qu'ils représentent, utiles par les liens qu'ils affichent ou les services documentaires qu'ils peuvent fournir. Dans le cas des institutions, centres et projets de recherche, les choix sont sans doute subjectifs et mentionnent des sites qui peuvent aussi intéresser par leur présentation.

\section{Sites généraux}

2 - Intute. Arts and Humanities

\section{www.intute.ac.uk/artsandhumanities}

Intute, portail de sites internet offrant des ressources pour l'éducation et la recherche, a été créé par un réseau d'institutions académiques et scientifiques du Royaume-Uni. La catégorie Arts and Humanities (à coté de Sciences and Technology, Social Sciences, Health and Life Sciences) est elle-même divisée en plusieurs sous-catégories, permettant ainsi de chercher parmi les 18000 sites référencés, qui font tous l'objet d'un commentaire pertinent sur leur but, leur contenu et leur utilité. La plupart des sites mentionnés sont anglo-saxons.

3 - The British Academy. The national academy for the humanities and the social sciences

\section{www.britac.ac.uk}

Ce site est surtout utile par les liens qu'il propose: les associations et institutions britanniques en sciences humaines (avec à chaque fois une description de 4-5 lignes, leurs coordonnées et un lien vers leur site); un répertoire des académies et des instituts de recherche britanniques à l'étranger (The British School à Athènes...) ; les projets soutenus par la British Academy (Corpus of Anglo-Saxon Stone Sculpture; Correspondance of James McNeill Whistler...).

4 - Association of Art Historians

\section{http://aah.org.uk}

Cette association professionnelle, née en 1974, a été fondée pour promouvoir et aider 
les études en histoire de l'art, conçue au sens large. Elle regroupe à la fois les étudiants, les enseignants, les personnels scientifiques des musées et des chercheurs indépendants (que l'on peut retrouver par mot-clés dans le répertoire en ligne). Le site présente les multiples activités de cette association ainsi que son fonctionnement. La liste des liens en ligne est particulièrement utile pour les arts visuels, le cinéma, la photographie et l'art contemporain.

\section{Bibliothèques et bibliographie}

5 - Londres, The British Library

www.bl.uk

Site essentiellement axé sur les collections de la British Library, pauvre en liens externes (que ce soit vers des documents numériques ou d'autres sites), mais remarquable pour les recherches dans les collections de la British Library d'autant plus que les moteurs de recherche, au lieu d'être uniques pour l'ensemble des différents objets de la collection (de la photographie au journal), sont adaptés à chaque support : possibilité ainsi pour la photographie de chercher par techniques (qui sont précisément décrites). De plus, les notices sont assez détaillées.

6 - Copac

http://copac.ac.uk

Copac est un catalogue unifié en ligne des 28 bibliothèques membres du CURL (Consortium des bibliothèques universitaires de recherches), qui inclut, à côté des bibliothèques d'Oxford et de Cambridge ou du Trinity College de Dublin, la British Library et la V\&A National Art Library. Il permet donc de faire des recherches (par auteur, mot du titre, date de parution - avec fourchette chronologique -, sujet...) dans un fichier total de 32 millions d'ouvrages. Les résultats peuvent être classés selon différents paramètres, et téléchargés. Un catalogue spécial a été réalisé pour les cartes.

7 - Oxford, The Bodleian Library

www.bodley.ox.ac.uk

Le site permet de chercher dans le catalogue de la Bodleian Library et dans les fonds des différentes bibliothèques de l'université d'oxford (Olis). Une de ses qualités est l'existence de liens internes qui permettent, à partir d'une première recherche, de trouver les autres publications du même auteur ou sur le même sujet.

8 - The English Short Title Catalogue (ESTC)

\section{http://estc.bl.uk}

Catalogue en ligne des ouvrages et périodiques imprimés avant 1801 dans les anciens territoires du royaume britannique (îles Britanniques, Canada..., mais aussi les ÉtatsUnis de 1776 à 1800) quelle que soit la langue, recensés dans plus de 20000 bibliothèques (British Library, Huntington Library...). Le moteur de recherche, très perfectionné, est le même que celui de la British Library qui héberge le site : il permet ainsi de rechercher tous les titres contenant tel mot imprimé entre telle date et telle date dans tel pays ou ville.

9 - Arlis

\section{www.arlis.net}

Arlis est une base de données en ligne concernant les périodiques en histoire de l'art (au sens large). Réalisée sous l'auspice de la National Art Library au V\&A Museum pour l'Art Library Society, cette base de données recense environ 18000 publications 
périodiques (du magazine aux collections) et indique leurs différentes localisations (et l'état des fonds) dans près de 250 bibliothèques ou institutions, tant publiques (Crafts Council, The Henry Moore Institute...) que privées (Bridgeman Art Library...) réparties dans le Royaume-Uni et en Irlande. Pour chacune de ces institutions, une fiche assez complète donne à la fois des renseignements pratiques, l'importance et les particularités du fonds en histoire de l'art, ainsi que les différents services proposés. Une page de documentation sur d'autres ressources documentaires tant au RoyaumeUni que dans le monde, avec liens, est particulièrement utile pour les catalogues de vente.

10 - The National Art Library

www.vam.ac.uk/nal

The Victoria and Albert Museum (V\&A Museum) héberge la National Art Library. Le catalogue en ligne (750 000 ouvrages) permet d'interroger également certains fonds particulièrement riches, comme celui des manuscrits ou des catalogues de vente. Les rubriques «Guides» et «Finding Information» offrent des bibliographies et des répertoires de sites internet ou d'autres informations particulièrement utiles, aussi bien sur Daniel Libeskind ou les papiers peints (rubrique «Guides») que sur les catalogues de vente ou les dictionnaires d'artistes britanniques (rubrique « Finding Information »).

11 - Bibliothèques publiques du Royaume-Uni

http://dspace.dial.pipex.com/town/square/ac940/weblibs.html

Portail qui renvoie non seulement aux sites des bibliothèques municipales et à leur catalogue quand il est en ligne, mais également à des sites plus généraux et utiles notamment au sein des bibliothèques de Londres, le catalogue collectif du M25 (Consortium de 25 [aujourd'hui 58] bibliothèques de recherche de Londres et ses environs).

\section{Archives et manuscrits}

12 - The National Archives

\section{www.nationalarchives.gov.uk}

Ce site, très anglais par sa convivialité et son côté "famille», offre la possibilité d'interroger l'ensemble des archives publiques sur le territoire du Royaume-Uni (par noms, mots-clés, en sélectionnant dans les fonds d'archives ou par fourchette chronologique). Le site détaille l'ensemble des catalogues mis en ligne et fournit d'utiles renseignements sur les outils de recherche dans les archives publiques et notamment, par le biais d'ARCHON Directory, sur les différentes institutions abritant des archives ou des manuscrits, avec des informations sur les catalogues et un lien vers leurs sites.

13 - Artists' Papers Register

\section{www.apr.ac.uk/artists/home.htm}

Catalogue en ligne des manuscrits et archives d'artistes, "designers» et artisans, conservés dans près de 1600 institutions (archives [dont le Public Record office], bibliothèques, musées, sociétés...). Une des forces de cet outil est la richesse des interrogations possible : par créateur ou société (William Morris, Morris \& Co Ltd), par lieu de conservation, mais aussi par catégorie d'activité (par exemple, pour la lettre T : tapissiers, historiens du textile, trustees...), par mots-clés (type de document, lieu 
géographique...). Un guide en ligne permet de bien orienter les recherches. La plupart des documents référencés sont postérieurs à 1800 .

\section{Dictionnaire biographique}

14 - Oxford Dictionary of National Biography

\section{www.oup.com/oxforddnb}

L'édition en ligne a permis une mise à jour des 33 volumes de l'oxford Dictionary of National Biography (2004). Elle comprend ainsi 56000 entrées biographiques et des listes de références et est abondamment illustrée. La consultation en ligne est payante, mais la plupart des grandes bibliothèques du Royaume-Uni y ont souscrit (la liste des bibliothèques où l'édition en ligne est disponible est consultable sur le site, mais la National Art Library n'est pas indiquée, alors qu'elle indique disposer du catalogue en ligne).

\section{Musées et Patrimoine}

15 - Royal Collection

\section{www.royalcollection.org.uk}

3140 notices avec images, des livres anciens aux éventails, incluant les peintures, dessins et les sculptures, mais aussi des vues des résidences royales; notices détaillées, moteur de recherche perfectionné, possibilité de zoomer dans les images.

16 - English Heritage

\section{www.english-heritage.org.uk}

Ce site recense les 400 sites et monuments dont la gestion est confiée à l'English Heritage, avec des fiches détaillées (pratiques et historiques) pour chaque lieu. De plus, la rubrique « Learning and Resources » offre différentes bases de données, dont :

- Images of England, site avec près de 370000 vues prises entre 1996 et 2005 pour environ 250000 bâtiments - du monument historique à la simple maison ancienne des 46 comtés d'Angleterre. Chaque bâtiment bénéficie d'une notice plutôt détaillée. À noter : la campagne photographique a été faite par des volunteers...

- Archeology Data Service: ce site donne l'accès à une carte archéologique de l'Angleterre (plus d'un million d'entrées...), avec pour chaque site un état des fouilles, des publications...

Pour les deux bases, les moteurs de recherches offrent de multiples possibilités de recherche, et il est possible de chercher par le biais d'une carte interactive.

- Londres, The British Museum

\section{www.thebritishmuseum.ac.uk}

Le site du British est principalement conçu pour le grand public. Il offre ainsi des fiches par civilisation et salle, et des notices illustrées de ses 4000 pièces importantes, avec un système de renvoi interne. Mais il contient également une partie recherche importante qui offre, outre des publications scientifiques en ligne (actes de journées d'études), des présentations de la cinquantaine de projets de recherche dans lesquels le British Museum est impliqué, avec parfois des ressources en ligne (Roman provincial coinage : une base de données concernant 13730 types monétaires, dont 9061 sont reproduits ; British printed images to 1700 , un site où sera mis prochainement en ligne 10000 
gravures antérieures à 1700). Comme beaucoup de sites anglais de musée, celui-ci présente les activités des conservateurs et fournit leur adresse électronique.

18 - Londres, The National Gallery

www.nationalgallery.org.uk

Le site offre en ligne le catalogue de la collection, avec pour chaque œuvre une notice détaillée et une explication en ligne des termes techniques à l'intention du grand public (predella...). Dans 378 cas, il est possible de zoomer sur le tableau, et des renvois internes sont proposés (le moteur de recherche, sommaire, permet néanmoins de chercher sur tous les mots de la notice). Le site Picture Library (www.nationalgalleryimages.co.uk) est spécifiquement destiné à commander des reproductions : il permet d'avoir une image plus fidèle de l'œuvre et avec une meilleure résolution mais les tableaux exposés n'appartenant pas à la National Gallery (les Sept sacrements de Poussin du Duc de Rutland, par exemple) ne sont pas présentés.

19 - Londres, The National Portrait Gallery

www.npg.org.uk

Le site, en plus de donner des renseignements pratiques sur ce musée, retrace son histoire, présente les activités du Heinz Archive and Library - centre de documentation pour le portrait - et d'autres programmes de recherche relatifs au portrait anglais (dont la base en ligne du 18th and 19th Century Caricatures and Portrait Prints), mais aussi sur la mezzotinte, les cadres .... Sur les 330000 œuvres de la National Portrait Gallery, près de 97000 sont en lignes, dont 51000 avec images. Le moteur de recherche, bien développé, permet de chercher par date, par fonds, par activité professionnelle ou pays du personnage portraituré, par types de portrait ou contenu de l'image (dans la catégorie "vêtement/gants »: 460 fiches) et des liens internes font circuler utilement au sein de la collection.

20 - Londres, Tate Gallery

\section{www.tate.org.uk}

65000 œuvres de la Tate Gallery (Britain et Modern) sont en ligne, avec pour chacune une notice élaborée. Mais le site de la Tate, un des plus réussis qui soit par son graphisme, offre également des cours audiovisuels en ligne, une série de fiches détaillées pour des problèmes de conservation (l'une d'elles, sur les cadres, est particulièrement bienvenue) et un catalogue en ligne de sa bibliothèque et de ses archives (qui inclut les documents d'acquisition des œuvres). Il offre également le bulletin scientifique de la Tate, Tate Papers (deux numéros par an, proposant chacun environ cinq articles).

21 - Londres, The Victoria and Albert Museum

\section{www.vam.ac.uk}

27000 objets de la collection sont en ligne, avec parfois des détails et des prises de vue sous différents angles (pas moins de 41 clichés pour Les trois Grâces de Canova, avec des images de leur disposition dans la salle). Un moteur de recherche développé permet d'interroger par technique, date ou recherche libre sur tous les termes de la notice.

22 - Catalogues de collections en ligne

Plusieurs musées offrent le catalogue de leurs collections en ligne, dont :

- Cambridge, Fitzwilliam Museum

www.fitzmuseum.cam.ac.uk

137000 objets, 102055 reproductions ; notices détaillées, moteur de recherche.

- Cardiff, National Museum Wales 
www.museumwales.ac.uk/en/home

2000 peintures et sculptures, avec reproductions; notices et moteur de recherche sommaire.

- Manchester, Manchester Art Gallery

www.manchestergalleries.org

30000 objets, pour la plupart reproduits ; notices détaillées ; moteur de recherche.

- Norfolk Museums and Archaeology Service

www.museums.norfolk.gov.uk

266000 objets en ligne, avec illustration, mais moteur de recherche et présentation peu pratiques.

- Oxford, Ashmolean Museum

www.ashmolean.org

Catalogue partiellement en ligne: les dessins français et italiens, les peintures...; notices avec images; moteur de recherche. À noter : le site internet de ce musée offre de plus des bases en ligne de recherche: Creswell Archive (6500 photographies d'architecture médiévale islamique), TileWeb (2 850 notices illustrées sur les carreaux émaillés du Moyen Âge)...

\section{Universités}

\section{- Universités au Royaume-Uni}

www.universitiesuk.ac.uk

Ce site officiel permet d'avoir une liste complète des universités. On peut la trouver également sous une forme plus dynamique sur hero (www.hero.ac.uk) ou, sous forme d'une carte interactive, sur www.scit.wlv.ac.uk/ukinfo

24 - Départements d'histoire de l'art

Il est généralement possible sur ces sites d'avoir, outre une présentation des cours, séminaires et programmes de recherche, un accès à la page personnelle de chaque enseignant.

- Aberdeen, University of Aberdeen, History of Art

www.abdn.ac.uk/hoart

- Birmingham, Birmingham University, Department of History of Art

www.historyofart.bham.ac.uk

- Bristol, University of Bristol, History of Art Department

www.bris.ac.uk/arthistory

- Cambridge, University of Cambridge, History of Art Department

www.hoart.cam.ac.uk

- Édimbourg, The University of Edinburgh, School of Art, Culture and Environment www.arthistory.ed.ac.uk

- East Anglia, University of East Anglia, School of World Art Studies and Museology

www1.uea.ac.uk/cm/home/schools/hum/worldart

- Essex, University of Essex, Art history Department

www2.essex.ac.uk/arthistory

- Glasgow, University of Glasgow, Department of History of Art

www.arthist.arts.gla.ac.uk

- Leeds, University of Leeds, History of Art and Cultural Studies

www.leeds.ac.uk/fine_art 
- Leicester, Leicester University, Department of History of Art and Film www.le.ac.uk/arthistory/welcome.html

- Londres, Birbeck University, Department of Art, Photography and Film www.bbk.ac.uk/hafvm

- Londres, Courtauld Institute of Arts

www.courtauld.ac.uk

- Londres, Goldsmith College, Visual Culture Department

www.goldsmiths.ac.uk/visual-cultures/index.php

- Londres, University College London, Art History Department

www.ucl.ac.uk/art-history/research/index.htm

- Londres, The Warburg Institute

http://warburg.sas.ac.uk

- Manchester, The University of Manchester, Art History and Visual Studies

www.arts.manchester.ac.uk/subjectareas/arthistoryvisualstudies

- Milton Keynes, The Open University, Department of History of Art

www.open.ac.uk/Arts/arthistory/index.html

- Nottingham, The University of Nottingham, Department of Art History

www.nottingham.ac.uk/art-history

- Oxford, Brookes University, Department of the History of Art

http://ah.brookes.ac.uk/arthistory

- Oxford, University of Oxford, History of Art Department

www.hoa.ox.ac.uk

- Reading, The University of Reading, Department of History of Art

www.arthistory.reading.ac.uk

- St Andrews, University of St Andrews, History of Art department

www-ah.st-andrews.ac.uk

- Sussex, University of Sussex, Art History Department

www.sussex.ac.uk/arthistory

- Warwick, The University of Warwick, History of Art

www2.warwick.ac.uk/fac/arts/arthistory

- York, The University of York, History of Art department

www.york.ac.uk/depts/histart/index.html

\section{Institutions, centres et projets de recherches}

\section{- A\&A. Art and Architecture}

www.artandarchitecture.org.uk

Cette base de photographies d'œuvres d'art, élégante mais peut-être un peu trop sobre dans sa présentation, est hébergée par le Courtauld Institute. Elle met en ligne 42000 images, qui sont les clichés de la Conway Library ou des peintures et dessins faisant partie de la Courtauld Gallery. Les points forts sont donc l'architecture et la sculpture (reproductions essentiellement en noir et blanc) et un de ses atouts est la qualité et la richesse des liens internes (par localisation, types de monuments, commanditaires, mots-clés) et la possibilité de se faire des dossiers (si on est enregistré sur le site).

- The Warburg Institute

http://warburg.sas.ac.uk

Le site du Warburg Institute en plus de présenter ses activités académiques (des 
séminaires aux publications), offre bien des ressources pour les chercheurs: un ensemble d'ouvrages numérisés et classés selon les thématiques propres à l'Institut (livres d'emblèmes, traités sur les antiquités de la période Renaissance et moderne,...), des bibliographies spécialisées, et un très précieux portail de bibliothèques en histoire des arts visuels.

- The British Archaeological Association

www.britarch.ac.uk/baa

Cette société savante fut créée en 1843 pour promouvoir les études en archéologie et histoire de l'architecture dans le domaine des antiquités nationales. Chaque année, elle offre des bourses et publie The Journal of the British Archeological Association ainsi qu'un volume des «Conferences » qui se tiennent tous les ans dans une ville différente. Le site se limite à présenter les activités de cette association.

- Le Corpus Vitrearum Medii Aevi (CVMA)

www.cvma.ac.uk

Le site britannique du CVMA, hébergé par le Courtauld Institute, offre des fiches de conservation pour les vitraux et prochainement des publications en ligne. Il inclut également une base de données de 18000 images de vitraux, pour la plupart en couleur, auxquelles on accède soit grâce à un moteur de recherche assez complet, soit par une carte interactive. Pour chaque œuvre, une fiche assez précise est disponible.

29 - Exeter Cathedral

http://hds.essex.ac.uk/exetercath

Ce site, réalisé par deux chercheurs, propose une base de données avec toutes les sculptures intérieures de la cathédrale d'Exeter. En plus d'un essai général, il est possible de détailler sur le plan les parties de l'édifice et les sculptures, chacunes faisant l'objet d'une notice détaillée.

30 - Center for the Study of the Renaissance at Warwick University

\section{www2.warwick.ac.uk/fac/arts/ren}

Ce centre de recherches pluridisciplinaire met en ligne une liste de ses membres, des informations sur les projets de recherches passés et en cours, et ses activités actuelles.

31 - Resources and Techniques for the Study of Renaissance and Early Modern Culture www2.warwick.ac.uk/fac/arts/ren/warburgwarwick

Co-dirigé par Charles Hope (Warburg) et Jonathan Davies (University of Warwick, Centre for the Study of the Renaissance), ce centre de recherches propose un enseignement spécialisé pour les doctorants travaillant sur la Renaissance et le début de la période moderne.

32 - Church Plans On-Line

\section{www.churchplansonline.org}

Cette base met en ligne les archives de la Incorporated Church Building Society, une institution fondée en 1818 pour rassembler des fonds afin de construire ou agrandir des églises anglicanes en Angleterre et dans le pays de Galles. Ces archives, actuellement conservées au Lambet Palace Library (siège des archives pour l'Église d'Angleterre) contiennent 15000 dossiers pour des projets de 1818 à 1982, avec environ 13000 plans qui ont été numérisés. Le moteur de recherche, perfectionné (il permet de sélectionner les projets non retenus, ceux d'un cabinet spécifique d'architectes,...) donne accès à une fiche concernant chaque projet, avec reproduction des plans ou des gravures anciennes, quand les documents existent. 
33

\section{www.victorianweb.org}

Un site plutôt grand public, fait par des anglicistes sur tous les aspects de la civilisation victorienne. Il est organisé en différents grands thèmes (philosophie, religion, science, arts visuels) eux mêmes structurés en différentes rubriques, qui contiennent pour la plupart des reproductions de textes imprimés sur ce sujet.

34 - The Préraphaelite Critic. Periodical criticism of the Preraphaelite movement: 1846 to 1900

\section{www.engl.duq.edu/servus/PR_Critic}

Sur ce site réalisé par un chercheur, on trouve notamment une abondante bibliographie d'articles concernant le mouvement et les artistes préraphaélites entre 1846 et 1860 , avec souvent un lien vers une transcription de ces articles.

35 - Oxford Portraits

\section{www.odl.ox.ac.uk/oxfordportraits}

Catalogue en ligne des portraits conservés à l'Université d'Oxford, en partie en libre accès. Pour chaque œuvre, est fournie outre les différentes rubriques habituelles (dimension, techniques, bibliographie, etc...) une longue description, une biographie de la personne représentée, et une transcription des documents concernant le portrait.

36 - The AHRB Research Centre for Studies of Surrealism and its Legacies www.surrealismcentre.ac.uk

Les universités d'Essex, de Manchester et la Tate Gallery sont les trois partenaires de ce centre de recherches. Son site présente les activités du centre, des publications et donne accès à l'intégralité des articles de sa revue Papers of Surrealism. Il met également à la disposition des chercheurs une base de données de 400 objets liés au surréalisme et conservés au Royaume-Uni.

\section{INDEX}

Index géographique : Grande-Bretagne

Mots-clés : Base de données

Keywords : Database 